\title{
Síndrome de burnout em profissionais de saúde antes e durante a pandemia da COVID-19
}

\author{
Burnout syndrome in healthcare professionals before and during the COVID-19 pandemic \\ Síndrome de burnout en profesionales de la salud antes y durante la pandemia de \\ COVID-19
}

Larissa Maciel Ribeiro ${ }^{1}$, Thayana de Almeida Vieira ${ }^{1}$, Karytta Sousa Naka² ${ }^{2 *}$

\begin{abstract}
RESUMO
Objetivo: Revisar a produção científica nacional sobre a magnitude dos efeitos físicos e mentais da Síndrome de Burnout (SB) em profissionais de saúde, com ênfase na comparação das características antes e durante a pandemia da COVID-19. Métodos: Trata-se de uma revisão integrativa da literatura, efetivada por artigos originais e completos publicados nas bases de dados: SCIELO, LILACS e GOOGLE ACADÊMICO, em língua portuguesa, espanhola e inglesa, publicados entre 2015 a 2020. Resultados: Foram incluídos 15 artigos na amostra final do estudo. Observou-se que os profissionais de saúde são os mais propensos ao desenvolvimento da SB e que durante uma pandemia, como da COVID-19, os fatores deliberativos para o acometimento ou intensificação dos sintomas se tornam constantes no cotidiano, permeando em um estado grave de exaustão física e emocional que ocasiona no desenvolvimento e progressão da síndrome no decorrer do período. Considerações finais: O presente estudo constatou que a efetividade do trabalho de profissionais da saúde depende de seu bem-estar, portanto, a sua saúde mental é fundamental para a qualidade de vida, sendo necessário o maior enfoque nesta ciência para uma excelente assistência a população, principalmente em momentos de emergência.
\end{abstract}

Palavras-chave: Esgotamento profissional, Saúde mental, Pandemia.

\begin{abstract}
Objective: To review the national scientific production on the magnitude of the physical and mental effects of Burnout Syndrome (SB) in health professionals, with an emphasis on comparing characteristics before and during the COVID-19 pandemic. Methods: This is an integrative literature review, carried out by original and complete articles published in the databases: SCIELO, LILACS and GOOGLE ACADÊMICO, in Portuguese, Spanish and English, published between 2015 and 2020. Results: 15 articles in the final study sample. It was observed that health professionals are the most prone to the development of BS and that during a pandemic, such as COVID-19, the deliberative factors for the onset or intensification of symptoms become constant in daily life, permeating a serious state of physical and emotional exhaustion that causes the development and progression of the syndrome over the period. Final considerations: The present study found that the effectiveness of the work of health professionals depends on their well-being, therefore, their mental health is fundamental to the quality of life, requiring a greater focus on this science for an excellent assistance to the population, especially in times of emergency.
\end{abstract}

Keywords: Professional exhaustion, Mental health, Pandemic.

\footnotetext{
${ }^{1}$ Faculdade Estácio de Castanhal (Estácio | FCAT), Castanhal - PA.

2 Instituto Evandro Chagas (IEC/SVS/MS), Ananindeua - PA.

*E-mail:karyttasousa@hotmail.com
} 


\section{RESUMEN}

Objetivo: Revisar la producción científica nacional sobre la magnitud de los efectos físicos y mentales del Síndrome de Burnout (SB) en profesionales de la salud, con énfasis en comparar características antes y durante la pandemia de COVID-19. Métodos: Se trata de una revisión bibliográfica integradora, realizada por artículos originales y completos publicados en las bases de datos: SCIELO, LILACS y GOOGLE ACADÊMICO, en portugués, español e inglés, publicados entre 2015 y 2020. Resultados: 15 artículos en la muestra final del estudio. Se observó que los profesionales de la salud son los más propensos al desarrollo de SB y que durante una pandemia, como la COVID-19, los factores deliberativos para la aparición o intensificación de los síntomas se vuelven constantes en la vida diaria, permeando un estado grave de Agotamiento físico y emocional que provoca el desarrollo y progresión del síndrome a lo largo del período. Consideraciones finales: El presente estudio encontró que la efectividad del trabajo de los profesionales de la salud depende de su bienestar, por lo tanto, su salud mental es fundamental para la calidad de vida, requiriendo un mayor enfoque en esta ciencia para una excelente asistencia a la población, especialmente en tiempos de emergencia.

Palabras clave: Agotamiento profesional, Salud mental, Pandemia.

\section{INTRODUÇÃO}

A pandemia da Coronavirus Disease 2019 (COVID-19), ocasionada pelo novo coronavírus, causador da Síndrome Respiratória Aguda Grave 2 (SARS-CoV-2), foi reportado inicialmente em dezembro de 2019, com origem na Província de Whuan, na China. A COVID-19 se espalhou gradativamente por diversos países, gerando impactos globais na área da saúde e grande preocupação na saúde mental e física nos profissionais de saúde. Devido o desconhecimento da doença e alta transmissibilidade do vírus, muitas deficiências foram evidenciadas na saúde pública com o aflorar do novo cenário (WHO, 2020).

Segundo Brooks SK, et al. (2020) a pandemia causa sequelas na saúde mental, ultrapassando até as mortes provocadas pela enfermidade. Os sistemas de saúde dos países atingidos entraram em decadência e os profissionais de saúde se tornam cada vez mais exaustos e afetados com as longas horas de trabalho e o distanciamento social - método de controle mais efetivo e adotado para reduzir a disseminação da doença. É notório que, em decorrência dessa perspectiva, o profissional tende a desenvolver um desgaste físico e emocional, além do surgimento de problemas como: hipertensão arterial, náuseas, estresse, doenças entéricas, esgotamento mental, depressão, exaustão e sono prejudicado.

A ocorrência dessas alterações está intrinsicamente vinculada ao trabalho, ainda mais nesta situação de surto. A Síndrome de Burnout (SB), também conhecida como síndrome do esgotamento profissional e psíquico, trata-se de uma doença de ordem psicossocial que aparece como resposta aos estressores interpessoais de natureza crônica que estão relacionados ao meio laboral, representando o maior índice de problema psicossocial e de estresse labora I(FERREIRA NN e LUCCA SR, 2015).

De acordo com Silveira ALP, et al. (2016) esta síndrome é ocasionada por uma combinação de três fatores: 1) Exaustão emocional (EE): caracterizada pela carência de Adenosina Trifosfato (ATP), motivação e empobrecimento sentimental que o próprio indivíduo não sabe lidar com seus afazeres diários e profissionais; 2) Despersonalização (DE): tende a provocar o desgaste na relação interpessoal, permitindo o olhar ao outro como um objeto que pode desencadear apatia e 3) Baixa realização profissional (RP): que condiz com a insatisfação individual e profissional que se opõe a negatividade frente ao ambiente ao qual se encontra.

Estas alterações comportamentais estão interligadas fisiologicamente, uma vez que há a diminuição de serotonina, ocitocina, dopamina e endorfina, os chamados "hormônios da felicidade" que são característicos deste déficit no indivíduo. Durante o decorrer da vida, o ser humano constantemente interage uns com os outros, o que os deixa mais expostos as situações de extenuação, tensão e inquietação, ocasionando comprometimento da saúde mental e física, principalmente, pelo estado de estresse e extrema tensão relacionado ao trabalho. Ademais, a exaustão emocional e a despersonalização, reflete graves efeitos sociais e interpessoais, além de se intensificar gradativamente no âmbito profissional (MACHADO PGB e PORTOMARTINS PC, 2015). 
Para Liang T (2020) a recente pandemia da COVID-19 representa um grande desafio no cenário atual por conta da calamidade em que se encontra a saúde global. Os profissionais de saúde que colaboram para o combate ao vírus altamente infeccioso, enfrentam inúmeros riscos de contaminação, falta de equipamentos pessoal e ausência de recursos materiais suficientes para garantir uma assistência à grande demanda de pacientes infectados.

Esses fatores resultam em impacto direto na saúde mental destes profissionais que permanecem em contato direto com a população doente, mesmo diante do constante de contrair a infecção e propagar o agente patogênico, o que acaba contribuindo para a deterioração da qualidade de vida desta categoria (LIMA DS, et al., 2020; CABELLO IR e PÉREZ IR, 2020).

O estabelecimento da SB se tornou ainda mais propício com a COVID-19, visto que a situação que já era desfavorável na saúde, agravou-se com o aumento das horas de trabalho. Os profissionais de saúde são ainda mais vulneráveis ao Burnout, pois apresentam diversos distúrbios psicológicos e osteomusculares recorrentes aos embates do âmbito profissional, além de lidarem com um ambiente de trabalho sem apoio social, déficit de recursos e cobrança rotineira para atender as demandas de infectados e as necessidades da população por eles atendida, o que pode configurar em um gatilho para o desencadeamento ou a intensificação do desgaste físico e mental (BAO Y, et al., 2020).

Assim, a SB tem gerado preocupação, pois tem sido considerada como um problema de saúde pública, uma vez a que sua incidência tem aumentado expressivamente nos últimos anos em diversos países, especialmente, no Brasil (SILVEIRA ALP, et al., 2016). A relevância da crise causada pela pandemia da COVID-19 gera alto índice de perturbações psíquicas e sociais tanto na população quanto nos profissionais de saúde, inibindo quaisquer resistências de capacidade em razão da sua magnitude (BRASIL, 2020).

Portanto, com o colapso na saúde, devido os efeitos da pandemia, no que condiz aos profissionais de saúde no enfrentamento da pandemia de COVID-19, a falta de amparo social, a ocupação laboral e o atendimento prestado a população nas instituições de saúde, geram alto índice de ansiedade e adoecimento, evoluindo para agravos a saúde, principalmente, no que diz respeito à saúde mental, como o aumento considerável da Burnout (ORGANIZAÇÃO MUNDIAL DA SAÚDE, 2020).

Diante deste contexto, a partir da compreensão que os profissionais de saúde tendem a sofrer com as condições de trabalho, as quais influenciam significativamente tanto na qualidade de vida e ocupação quanto na potencialidade da assistência prestada à população nas instituições de saúde, o presente estudo objetivou revisar a produção científica nacional sobre a magnitude dos efeitos físicos e mentais da Síndrome de Burnout em profissionais de saúde, com ênfase na comparação das características antes e durante a pandemia da COVID-19.

\section{MÉTODOS}

Trata-se de uma revisão integrativa da literatura no que se refere à SB e seus impactos em profissionais da saúde e o contexto da pandemia da COVID-19, baseado nas seguintes etapas: 1) elaboração da pergunta da revisão; 2) busca e seleção dos estudos primários; 3) extração de dados dos estudos; 4) avaliação crítica dos estudos primários incluídos na revisão; 5) síntese dos resultados da revisão e 6) apresentação do método (MENDES KDS, et al., 2019).

Foram utilizadas as seguintes bases de dados: Literatura Latino-Americana e do Caribe em Ciências da Saúde (LILACS), Biblioteca Eletrônica Científica Online (SCIELO) e Google acadêmico. A busca foi realizada nos idiomas português, espanhol e inglês e norteada com a associação dos termos extraídos do Descritores em Ciências da Saúde (DeCS): "Esgotamento Profissional", "Saúde Mental" e "Pandemia".

Os termos foram combinados de diferentes formas por meio do operador booleano "AND" para garantir uma busca ampla e responder à pergunta norteadora. Os critérios de inclusão adotados foram: estudos publicados no período de 2015 a 2020; textos completos disponíveis online e nos idiomas português, espanhol e inglês que abordassem sobre a temática. Foram excluídas teses e dissertações, artigos de relatos de caso ou experiência, cartas, editoriais e artigos científicos duplicados entre as bases de dados. 
O tema definido na pesquisa foi a viabilidade do estresse e Síndrome de Burnout em profissionais de saúde em virtude do cenário de pandemia da COVID-19, com base na seguinte questão de pesquisa: Quais os principais achados da literatura nacional relacionados a síndrome de Burnout em profissionais de saúde? Qual a incidência da SB nestes profissionais de saúde antes e durante o combate à COVID-19?

Figura 1 - Fluxograma da estratégia de busca dos artigos.

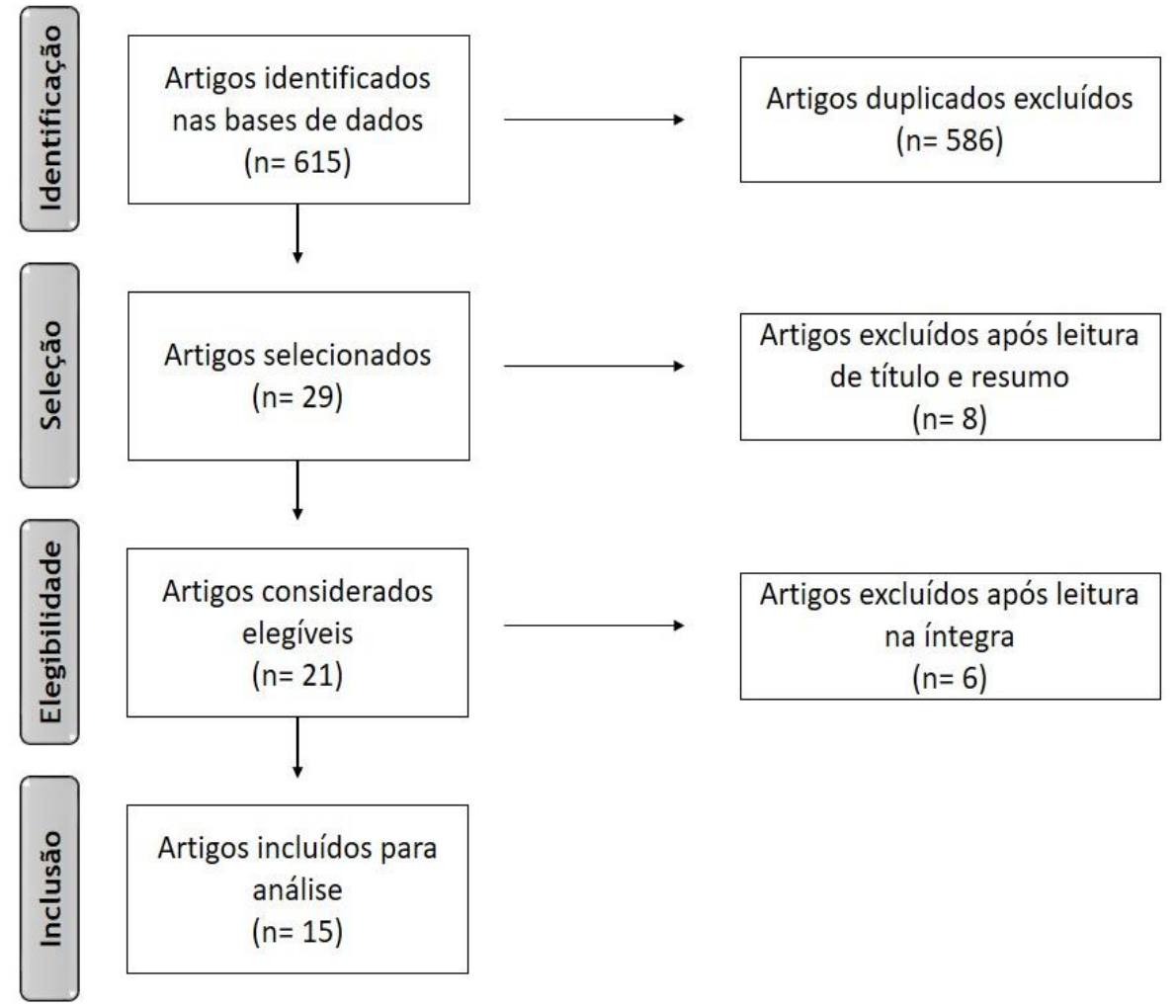

Fonte: Ribeiro LM, et al., 2020.

\section{RESULTADOS}

Com referência ao ano de publicação, notou-se que do total de 15 artigos selecionados, o ano de 2020 apresentou 60\% das publicações no período analisado, seguido dos anos de 2018 e 2019, com 13,3\%. O ano de 2016 não obteve estudos selecionados para a revisão (Tabela 1).

Tabela 1 - Distribuição dos artigos conforme o ano de publicação.

\begin{tabular}{ccc}
\hline Ano de publicação & N & $\%$ \\
\hline 2015 & 1 & 6,6 \\
2016 & 0 & 0,0 \\
2017 & 1 & 6,6 \\
2018 & 2 & 13,3 \\
2019 & 2 & 13,3 \\
2020 & 9 & 60,0 \\
\hline Total & $\mathbf{1 5}$ & $\mathbf{1 0 0 , 0}$
\end{tabular}

Fonte: Ribeiro LM, et al., 2020.

A partir dos artigos selecionados foi realizada a distribuição dos estudos de acordo com a autoria, ano de publicação, título, periódico, objetivo do estudo e base de dados (Quadro 1), a fim de apresentar a síntese dos resultados encontrados nesta revisão. 
Quadro 1 - Distribuição dos estudos selecionados de acordo com a autoria, ano de publicação, título, periódico, objetivos do estudo e base de dados. Castanhal-PA, 2020.

\begin{tabular}{|c|c|c|c|c|c|}
\hline $\mathbf{N}$ & Autores e ano & Título & Periódico & Objetivo & $\begin{array}{c}\text { Base de } \\
\text { dados }\end{array}$ \\
\hline 1 & $\begin{array}{l}\text { DINIZ LFM, et } \\
\text { al., (2020) }\end{array}$ & $\begin{array}{l}\text { Saúde mental na pandemia de } \\
\text { COVid-19: Considerações práticas } \\
\text { multidisciplinares sobre cognição, } \\
\text { emoção e comportamento. }\end{array}$ & $\begin{array}{l}\text { Debates em } \\
\text { psiquiatria }\end{array}$ & $\begin{array}{l}\text { Apontar as contribuições da psicologia e de ciências } \\
\text { relacionadas que possam auxiliar no entendimento e manejo } \\
\text { do comportamento humano frente à pandemia de COVID- } 19 \text {. }\end{array}$ & SCIELO \\
\hline 2 & $\begin{array}{l}\text { PEREIRA MD, } \\
\text { et al., (2020) }\end{array}$ & $\begin{array}{l}\text { Sofrimento emocional dos } \\
\text { enfermeiros no contexto hospitalar } \\
\text { frente à pandemia de COVID-19 }\end{array}$ & $\begin{array}{l}\text { Research, Society } \\
\text { and Development }\end{array}$ & $\begin{array}{l}\text { Realizar uma reflexão teórica sobre o sofrimento emocional } \\
\text { dos enfermeiros no contexto hospitalar frente à pandemia de } \\
\text { COVID-19. }\end{array}$ & $\begin{array}{c}\text { Google } \\
\text { Acadêmico }\end{array}$ \\
\hline 3 & $\begin{array}{l}\text { SCHMIDT B, } \\
\text { et al., (2020) }\end{array}$ & $\begin{array}{c}\text { Impactos na Saúde Mental e } \\
\text { Intervenções Psicológicas Diante da } \\
\text { Pandemia do Novo Coronavírus } \\
\text { (COVID-19) } \\
\end{array}$ & $\begin{array}{l}\text { Estudos de } \\
\text { Psicologia }\end{array}$ & $\begin{array}{l}\text { Sistematizar conhecimentos sobre impactos na saúde mental } \\
\text { e intervenções psicológicas diante da pandemia do novo } \\
\text { coronavírus. }\end{array}$ & SCIELO \\
\hline 4 & $\begin{array}{l}\text { LATORRACA } \\
\text { COC, et al., } \\
(2019)\end{array}$ & $\begin{array}{l}\text { O que as revisões sistemáticas } \\
\text { Cochrane dizem sobre prevenção e } \\
\text { tratamento da síndrome de burnout e } \\
\text { estresse no trabalho. }\end{array}$ & Diagn Tratamento & $\begin{array}{l}\text { Apresentar as evidências de revisões sistemáticas (RSs) } \\
\text { Cochrane sobre eficácia e segurança das estratégias de } \\
\text { tratamento e prevenção da síndrome de burnout. }\end{array}$ & LILACS \\
\hline 5 & $\begin{array}{l}\text { RAMOS RS, } \\
\quad(2020)\end{array}$ & $\begin{array}{l}\text { A Enfermagem Oncológica no } \\
\text { Enfrentamento da Pandemia de } \\
\text { Covid-19: Reflexões e } \\
\text { Recomendações para a Prática de } \\
\text { Cuidado em Oncologia }\end{array}$ & $\begin{array}{l}\text { Revista Brasileira } \\
\text { de Cancerologia }\end{array}$ & $\begin{array}{l}\text { Apresentar uma reflexão sobre as práticas de cuidado da } \\
\text { enfermagem no contexto da oncologia em tempos do } \\
\text { enfrentamento da pandemia da Covid-19 no Brasil }\end{array}$ & $\begin{array}{l}\text { Google } \\
\text { Acadêmico }\end{array}$ \\
\hline 6 & $\begin{array}{l}\text { OLIVEIRA RF, } \\
\text { et al., (2017) }\end{array}$ & $\begin{array}{l}\text { Incidência da síndrome de burnout } \\
\text { nos profissionais de enfermagem: } \\
\text { uma revisão integrativa }\end{array}$ & $\begin{array}{l}\text { Revista de } \\
\text { Enfermagem do } \\
\text { Centro-Oeste } \\
\text { Mineiro }\end{array}$ & $\begin{array}{c}\text { Explorar as produções científicas disponíveis na literatura } \\
\text { analisando a incidência da Síndrome de Burnout nos } \\
\text { profissionais da área da enfermagem. }\end{array}$ & LILACS \\
\hline 7 & $\begin{array}{l}\text { LARRÉ MC, et } \\
\text { al., (2018) }\end{array}$ & $\begin{array}{l}\text { A relação da síndrome de Burnout } \\
\text { com os profissionais de enfermagem; } \\
\text { revisão integrativa }\end{array}$ & Revista Nursing & $\begin{array}{l}\text { O objetivo do estudo consiste em identificar a ocorrência da } \\
\text { síndrome de Burnout no profissional de enfermagem. }\end{array}$ & LILACS \\
\hline
\end{tabular}

REAS/EJCH | Vol.12(11) | e5021 | DOI: https://doi.org/10.25248/reas.e5021.2020 Página 5 de 10 
Revista Eletrônica Acervo Saúde / Electronic Journal Collection Health ｜ ISSN 2178-2091

\begin{tabular}{|c|c|c|c|c|c|}
\hline $\mathbf{N}$ & Autores e ano & Título & Periódico & Objetivo & $\begin{array}{l}\text { Base de } \\
\text { dados }\end{array}$ \\
\hline 8 & $\begin{array}{l}\text { HUMEREZ } \\
\text { DC, et al., } \\
(2020)\end{array}$ & $\begin{array}{l}\text { Saúde mental dos profissionais de } \\
\text { enfermagem do brasil no contexto da } \\
\text { pandemia covid-19: ação do } \\
\text { conselho federal de enfermagem }\end{array}$ & $\begin{array}{l}\text { Cogitare } \\
\text { enfermagem }\end{array}$ & $\begin{array}{l}\text { Refletir sobre a saúde mental dos profissionais de } \\
\text { enfermagem do Brasil no contexto da pandemia COVID-19. }\end{array}$ & $\begin{array}{l}\text { Google } \\
\text { Acadêmico }\end{array}$ \\
\hline 9 & $\begin{array}{l}\text { SAIDEL MGB, } \\
\text { et al., (2020) }\end{array}$ & $\begin{array}{l}\text { Mental health interventions for health } \\
\text { professionals in the context of the } \\
\text { Coronavirus pandemic }\end{array}$ & $\begin{array}{l}\text { Revista } \\
\text { enfermagem } \\
\text { UERJ }\end{array}$ & $\begin{array}{c}\text { Refletir sobre intervenções / ações em saúde mental voltadas } \\
\text { aos profissionais de saúde que prestam assistência a } \\
\text { pacientes suspeitos ou diagnosticados com COVID-19. }\end{array}$ & LILACS \\
\hline 10 & $\begin{array}{l}\text { ENUMO SRF, } \\
\text { et al., (2020) }\end{array}$ & $\begin{array}{l}\text { Enfrentando o estresse em tempos } \\
\text { de pandemia: Proposição de uma } \\
\text { cartilha }\end{array}$ & $\begin{array}{l}\text { Revista Estudos } \\
\text { de Psicologia }\end{array}$ & $\begin{array}{l}\text { Apresentar a possibilidade de elaboração de materiais } \\
\text { práticos/aplicados com base em estudos científicos no } \\
\text { contexto da Psicologia aplicada à área da saúde }\end{array}$ & LILACS \\
\hline 11 & $\begin{array}{l}\text { LIMA AS, et } \\
\text { al., (2018) }\end{array}$ & $\begin{array}{l}\text { Análise da prevalência da síndrome } \\
\text { de burnout em profissionais da } \\
\text { atenção primária em saúde }\end{array}$ & $\begin{array}{l}\text { Trabalho } \\
\text { Educação Saúde }\end{array}$ & $\begin{array}{l}\text { Identificar a prevalência da SB nos profissionais da APS e } \\
\text { fatores associados. }\end{array}$ & SCIELO \\
\hline 12 & $\begin{array}{l}\text { ZANATTA AB } \\
\text { e LUCCA SR, } \\
\qquad(2015)\end{array}$ & $\begin{array}{l}\text { Prevalência da síndrome de burnout } \\
\text { em profissionais da saúde de um } \\
\text { hospital oncohematológico infantil }\end{array}$ & $\begin{array}{l}\text { Revista da Escola } \\
\text { de Enfermagem } \\
\text { da USP }\end{array}$ & $\begin{array}{l}\text { Identificar a prevalência da SB em profissionais médicos, } \\
\text { enfermeiros e técnicos de enfermagem de um hospital } \\
\text { oncohematológico infantil no estado de São Paulo }\end{array}$ & SCIELO \\
\hline 13 & $\begin{array}{l}\text { BRITO TB, et } \\
\text { al., (2019) }\end{array}$ & $\begin{array}{c}\text { Síndrome de burnout: estratégias de } \\
\text { prevenção e tratamento nos } \\
\text { profissionais de enfermagem. }\end{array}$ & Revista UNINGÁ & $\begin{array}{l}\text { Analisar artigos referentes a tema identificando as estratégias } \\
\text { para prevenção e tratamento da SB. }\end{array}$ & LILACS \\
\hline 14 & $\begin{array}{l}\text { FARO A, et al., } \\
\qquad(2020)\end{array}$ & $\begin{array}{l}\text { COVID-19 e saúde mental: A } \\
\text { emergência do cuidado }\end{array}$ & $\begin{array}{l}\text { Estud. psicol. } \\
\text { (Campinas) } \\
\text { [online] }\end{array}$ & $\begin{array}{l}\text { Reunir informações e achados de pesquisa a respeito do } \\
\text { impacto de tais crises na saúde mental. }\end{array}$ & LILACS \\
\hline 15 & $\begin{array}{l}\text { ORTIZ JR, et } \\
\text { al., (2020) }\end{array}$ & $\begin{array}{l}\text { Consecuencias de la pandemia } \\
\text { covid-19 en la salud mental } \\
\text { asociadas al aislamiento social }\end{array}$ & $\begin{array}{l}\text { Asociación } \\
\text { Mexicana de } \\
\text { Psicoterapia y } \\
\text { Educación }\end{array}$ & $\begin{array}{l}\text { Mostre as possíveis consequências na saúde mental da } \\
\text { população que pode começar a ter sucesso como resultado do } \\
\text { conflito social devido à pandemia COVID-19. }\end{array}$ & SCIELO \\
\hline
\end{tabular}

Fonte: Ribeiro LM, et al., 2020.

REAS/EJCH | Vol.12(11) | e5021 | DOI: https://doi.org/10.25248/reas.e5021.2020 Página 6 de 10 


\section{DISCUSSÃO}

A síndrome de Burnout está em crescente prevalência no Brasil nos últimos anos. Segundo dados do International Stress Management Association no Brasil (ISMA-BR), no ano de 2019, $72 \%$ da população economicamente ativa do país possuíam altos níveis de estresse. Desses, $32 \%$ desenvolveram Burnout, com sinais e sintomas característicos. Isso ocorre em decorrência da alta competitividade do mercado e a falta de tratamento precoce dos sintomas por preconceito e outros paradigmas (LATORRACA COC, et al., 2019).

Foi possível observar por meio dos artigos selecionados que na SB, o profissional, em dado momento, desiste, perde a energia e o sentido de sua relação com o trabalho (BRASIL, 2019). De acordo com a Organização Mundial de Saúde (OMS), aproximadamente 10\% das faltas e afastamentos estão relacionados ao estresse vivenciado no ambiente de trabalho. No campo da saúde e antes da pandemia, a prevalência da SB se encontrava em cerca de $78,4 \%$ dos profissionais de saúde do Brasil. Além disso, é importante citar que a Burnout frequentemente pode ser confundida com a depressão, podendo apresentar dados mais alarmantes (SILVEIRA ALP, et al., 2016; OMS, 2019).

Antes da pandemia, estudos epidemiológicos sobre SB no Brasil se mostram escassos devido a definição relativamente recente do termo "síndrome de Burnout". Contudo, estima-se que haverá um aumento progressivo da SB no país. Comparando os períodos antes e durante a pandemia, a enfermidade tem presença marcante durante a pandemia, estabelecendo um problema de saúde pública por afetar profissionais de saúde e servidores gerais das entidades de saúde que permanecem na linha de frente de combate ao COVID-19. Este surto na saúde mental pode afetar diretamente as perspectivas e metas traçadas para a retomada econômica e para o fim da pandemia, sendo essencial o desenvolvimento de estratégias destinadas a fortalecer a saúde mental da população afetada (ORTIZ JM, et al., 2020).

A maioria dos países afetados pela pandemia da COVID-19 tem adotado o regime de quarentena para conter o contágio. Em situações pandêmicas, constatou-se que alguns transtornos mentais comuns podem ser desencadeados pela quarentena, como transtornos de ansiedade e depressão e indícios de aumento do comportamento suicida. Estes impactos negativos também propiciam o surgimento de problemas mentais nos profissionais de saúde devido alguns estressores, como: insegurança, melancolia, medo e desconhecimento do tempo de duração da pandemia, conjuntamente com a sobrecarga de trabalho (FARO A, et al., 2020).

O contexto da pandemia exigiu que o profissional reformulasse suas habilidades e competências, a fim de se adequar ao desenvolvimento de um cuidado que estivesse apto para suprir as demandas inerentes a cada profissão no momento atual. Contudo, essas condições específicas do cenário e a grande carga de trabalho e responsabilidade se caracterizaram como causadores de estresse laboral e fatores de risco ao comprometimento da saúde destes profissionais (RAMOS RS, 2020). Para Saidel MGB, et al. (2020) a pandemia da COVID-19 possibilitou identificar as diversas fragilidades em relação a saúde mental dos profissionais de saúde nos países afetados em tempos de crise, inclusive no Brasil.

Apesar do protagonismo vivenciado pelos profissionais de saúde frente a pandemia, a exposição e a pressão para equilibrar demandas profissionais e familiares, os sentimentos de medo, exaustão, ambivalência, o papel que desempenham de agente cuidador e a necessidade de atendimento imediato e especializado para uma significativa parcela de pessoas com sintomas da COVID-19, estes profissionais permanecem em um estado de cobrança e culpa permanentes em virtude de tentativas para conciliar as obrigações diárias (LIMA DS, et al., 2020; CABELLO IR e PÉREZ IR, 2020).

Assim, o desgaste gerado pela presença destes conflitos e os desafios enfrentados para zelar e preservar o exercício profissional de forma segura, compreendem um dos principais fatores desencadeantes da Burnout, sendo possível caracterizar estas condições como fatores distintivos para administrar a vida pessoal e profissional. Portanto, o sofrimento emocional gerado, além de levar a deterioração da qualidade de vida, também interfere na assistência prestada pelos profissionais de saúde (TEIXEIRA FD e PREBIANCHI HB, 2019).

Além disso, quando o profissional é sujeito à exaustão, pode desenvolver alterações cognitivas que afetam a organização dos pensamentos e comprometem a saúde imunológica, hormonal e cardíaca, resultando em dores musculares, deficiência no sono e transtornos sexuais. Assim, o excesso na rotina de trabalho e o 
comprometimento psicológico acabam por afetar a saúde integral e acelerar crescimento das taxas de absenteísmo, contribuindo para o aumento dos níveis de eventos indesejáveis como acidente laborais, distanciamento social e delimitação na realização das tarefas (LIMA AS, et al., 2018).

Nesse ponto de vista, o trabalho, a saúde e o adoecimento estão interligados à vida dos indivíduos, pois a atividade ocupacional reflete tanto na saúde mental quanto na física. Dessa maneira, ao mesmo tempo que o trabalho é uma fonte de prazer, também pode se tornar um gerador de sofrimento, seja em maior ou menor grau, podendo ser capaz de causar danos à saúde dos profissionais e, na área da saúde, isto não se difere (BARBOZA PC, et al., 2018; BAO Y, et al., 2020; LI Z, et al., 2020).

No cenário atual, Pereira MD, et al. (2020) evidenciam em seu estudo que vários sintomas de sofrimento foram identificados em profissionais de saúde e, em especial, nos de enfermagem devido ao ambiente laboral de alto risco de contaminação pelo vírus, efeito da doença na vida profissional e alta demanda de pacientes aos seus cuidados, os quais desencadeiam um sentimento de impotência e insegurança profissional, estando tendenciados a enfrentar situações estressantes e com necessidade de rápida tomada de decisão. Tal perspectiva induz a esse grupo populacional a prática da resiliência nas adversidades, contudo também leva a propensão ao desenvolvimento da SB (ORTIZ JM, et al., 2020).

A OMS observou que durante o colapso da saúde causada pela pandemia, os profissionais da enfermagem apresentaram altos níveis de ansiedade, adicionados ao risco de contagio, provocaram severos problemas de saúde mental e física, resultando no aumento de casos da Síndrome de Burnout, além de gerar ansiedade, depressão e estresse.

Segundo o Conselho Federal de Enfermagem (COFEN), no Brasil, o número de profissionais afastados no mês de abril de 2020 , aumentou $660 \%$, saltando de 158 para 1.203 enfermeiros que se ausentaram dos seus postos de trabalho (HUMEREZ DC, et al., 2020).

De acordo com Urquiza JLG, et al. (2016), o Brasil apresenta predomínio de sintomas depressivos em profissionais da enfermagem. Esta classe é a principal responsável pela prestação da assistência, estando em contato direto com o paciente e seus familiares. Logo, a falta de autonomia, a sobrecarga de trabalho e a má definição do papel desses profissionais fazem com que a enfermagem seja a classe mais susceptível ao surgimento da SB (LARRÉ MC, et al., 2018).

Verificou-se que os profissionais de saúde chineses que cuidavam diretamente de pacientes com a infecção, mostraram dados importantes relacionados à saúde mental. De um total de 1.257 profissionais que trabalham em 34 hospitais (39\% médicos e $61 \%$ enfermeiros), aproximadamente $50 \%$ apresentaram sintomas de depressão, $45 \%$ ansiedade, $34 \%$ de insônia e $72 \%$ de angústia.

Os sintomas foram maiores em enfermeiras, mulheres e profissionais que forneceram atendimento a pacientes com COVID-19 (LAI J, et al., 2020; SAIDEL MGB, et al., 2020). Tais dados, apesar de serem da China, reafirmam a constatação de que durante uma crise na área da saúde, como a atual, há a maior possibilidade que experiências negativas sejam vivenciadas de maneira excessiva pela população, permeando na necessidade de amparo psicológico, além de demonstrar possíveis situações que podem ocorrer no Brasil (HO C, et al., 2020; SCHMIDT B, et al., 2020).

O sofrimento gerado impacta tanto em relação ao desempenho profissional do indivíduo como nas questões econômica e social das instituições de saúde que prestam serviços essências em momentos como este (TEIXEIRA FD e PREBIANCHI HB, 2019). Fernandes MA, et al. (2018) salientam que o sofrimento prejudica vários âmbitos da vida dos trabalhadores, sejam eles: familiar, social, pessoal, profissional, acadêmica, autoconhecimento, a compreensão dos outros, a capacidade de autocrítica, aceitação de problemas e a possibilidade de ter prazer na vida.

A promoção da saúde mental tem sido o enfoque no âmbito hospitalar com o passar da pandemia. A percepção de que esta interfere no bem-estar e no desenvolvimento das potencialidades humanas, seja em qualquer profissional da saúde acometido pela SB ou outro problema psíquico, permitiu o emprego de estratégias de enfretamento para identificação de estressores que precipitam a severidade das enfermidades de modo a minimizar os impactos negativos da crise. 
Apesar das inúmeras adversidades e mudanças advindas do período, muitos profissionais não aceitam o tratamento preventivo, até progredir a um estado de exaustão extrema, permeando em diversas complicações, assim, não reconhecendo a necessidade de investigar e agir em questões relativas à saúde mental ao longo da crise (ENUMO SRF, et al., 2020).

É importante manter vigilância sobre os fatores que configuram risco para o estabelecimento da SB nos profissionais, tais como: as inadequadas condições de trabalho, pouco suporte social, baixa remuneração, não reconhecimento pelo trabalho realizado, longa carga horária, contato direto com pessoas em sofrimento físico e psíquico.

Para uma abordagem satisfatória, também é necessário considerar a vulnerabilidade e especificidade de cada indivíduo particularmente, a fim de contribuir com iniciativas sistematizadas pelas instituições de saúde no acolhimento de seus integrantes, mitigar as consequências negativas da pandemia e fomentar o bem-estar dos profissionais de saúde no âmbito da promoção e manutenção da saúde, prevenção e tratamento de doenças (MORRISON V e BENNETT P, 2016).

Este artigo teve como principal obstáculo a baixa produção científica que abordasse a relação da SB com a pandemia da COVID-19, buscando aspectos gerais para explicar esse fenômeno, sendo necessário a ampliação das pesquisas no Brasil para compreensão e melhoria das condições de trabalho dos profissionais de saúde.

\section{CONSIDERAÇÕES FINAIS}

A efetividade do trabalho de profissionais da saúde depende de seu bem-estar, portanto, a sua saúde mental é fundamental para a qualidade de vida da população por ele atendida. Foi possível verificar que os profissionais de enfermagem são mais acometidos pela SB. Ressalta-se que a pandemia é um fator de preocupação para o estabelecimento da SB, decorrente aos sentimentos de frustação, insegurança e exaustão que se originaram/originam durante o período. Nesse sentido, é relevante a avaliação e acompanhamento da saúde mental e física dos profissionais desta área, além da gestão apropriada do trabalho. Este artigo serve de alerta para um possível surto na saúde mental destes e outros profissionais que atuam excessivamente mesmo na pandemia, visto que uma vez que são negligenciados a longo prazo, podem evoluir de sintomas leves para quadros mais graves como a síndrome de Burnout.

\section{REFERÊNCIAS}

1. BAO Y, et al. 2019-nCoV epidemic: address mental health care to empower society. The Lancet, 2020; 395(10224): 37-38.

2. BARBOZA PC, et al. Significado do trabalho: perspectivas de profissionais de enfermagem atuantes em unidades clínicas. Revista Rene (Online), 2018; 1(1): 1-8.

3. BRASIL. Ministério da Saúde. 2019. Lista de doenças relacionadas ao trabalho. Portaria N1339 (elaborada em cumprimento da Lei 8.080/90).

4. BRASIL. Ministério da Saúde. 2020. Plano de contingência nacional para infecção humana pelo novo Coronavírus 2019-nCoV: Centro de operações de emergências em saúde pública (COE-nCoV).

5. BRITO TB, et al. Síndrome de burnout: estratégias de prevenção e tratamento nos profissionais de enfermagem. Revista UNINGÁ, 2019; 56(2): 113-122.

6. BROOKS SK, et al. The psychological impact of quarantine and how to reduce it: rapid review of the evidence. The Lancet, 2020; 395(102227): 912-20.

7. CABELLO IR, PÉREZ IR. (2020). El impacto de la pandemia por COVID-19 sobre la salud mental de los profesionales sanitarios. (Org.). Escuela Anadaluza de Salud Pública: Consejería de Salud y Familias - Espanha.

8. DINIZ LFM, et al. Saúde mental na pandemia de COVID-19: Considerações práticas multidisciplinares sobre cognição, emoção e comportamento. Debates em psiquiatria, 2020; ?(spec.): 1-23.

9. ENUMO SRF, et al. Enfrentando o estresse em tempos de pandemia: Proposição de uma cartilha. Revista Estudos de Psicologia, 2020; ?(spec.):1-35.

10. FARO A, et al. COVID-19 e saúde mental: A emergência do cuidado. Estud. psicol. (Campinas) [online], 2020; $37: 1$ 29.

11. FERNANDES MA, et al. Work-related mental disorders among nursing professionals: A Brazilian integrative review. Revista Brasileira de Medicina do Trabalho, 2018; 16(2): 218-224. 
12. FERREIRA NN, LUCCA SR. Síndrome de Burnout em técnicos de enfermagem de um hospital público do Estado de São Paulo. Revista brasileira de epidemiologia, 2015; 18(1): 68-79.

13. HENRIQUES SJ. Contributo da psicologia da saúde na promoção de comportamentos salutogénicos em pandemia. Psicologia, saúde \& doenças, 2020; 21(2): 297-310.

14. HO C, et al. Mental health strategies to combat the psychological impact of COVID-19 beyond paranoia and panic. Annals Academy Medical of Singapure, 2020; 49(3): 1-3.

15. HUMEREZ DC, et al. Saúde mental dos profissionais de enfermagem do brasil no contexto da pandemia covid-19: ação do conselho federal de enfermagem. Cogitare enfermagem, 2020; 25: 1-10.

16. LAI J, et al. Factors Associated With Mental Health Outcomes Among Health Care Workers Exposed to Coronavirus Disease 2019. JAMA Netw Open, 2020; 3(3): 1-12.

17. LARRÉ MC, et al. A relação da síndrome de Burnout como os profissionais de enfermagem: revisão integrativa. Revista Nursing, 2018; 21 (237): 2018-2023.

18. LATORRACA COC, et al. O que as revisões sistemáticas Cochrane dizem sobre prevenção e tratamento da síndrome de burnout e estresse no trabalho. Diagn Tratamento, 2019; 24(3): 119-125.

19. LI Z, et al. Vicarious traumatization: A psychological problem that cannot be ignored during the COVID-19 pandemic. Brain, Behavior, And Immunity,2020; 87: 74.

20. LIANG T. 2020. Zhejiang University School of Medicine. Handbook of COVID-19: Prevention and treatment. Paris:International Association of Universities, UNESCO.

21. LIMA AS, et al. Análise da prevalência da síndrome de burnout em profissionais da atenção primária em saúde. Trabalho Educação e Saúde, 2018; 16(1): 283-304.

22. LIMA DS, et al. Recommendations for emergency surgery during the COVID-19 pandemic. CJMB, 2020; 8(1): 1-3.

23. MACHADO PGB e PORTO-MARTINS PC. Condições organizacionais enquanto terceiras variáveis entre burnout e engagement. Diaphora, 2015; 13(1): 35-44.

24. MENDES KDS, et al. Uso de gerenciador de referências bibliográficas na seleção dos estudos primários em revisão integrativa. Texto Contexto Enfermagem, 2019; 28: 1-13.

25. MORENO JK, et al. Síndrome de Burnout e fatores de estresse em enfermeiros nefrologistas. Revista de Enfermagem UFPE On Line, 2018; 4 (12): 865-71.

26. MORRISON V, BENNETT P. What is health. In An Introduction to Health Psychology. 4th ed. London, United Kingdom: Pearson Education Limited, 2016; 24p.

27. OLIVEIRA RF, et al. Incidência da síndrome de burnout nos profissionais de enfermagem: uma revisão integrativa. Revista de Enfermagem do Centro-Oeste Mineiro, 2017; 7: 1-9.

28. ORGANIZAÇÃO MUNDIAL DA SAÚDE (OMS). 2019. Síndrome de burnout é detalhada em classificação internacional da OMS.

29. ORTIZ JR, et al. Consecuencias de la pandemia covid-19 en la salud mental asociadas al aislamiento social. Asociación Mexicana de Psicoterapia y Educación, 2020: 1-21.

30. PEREIRA BAMT. (Org.) Burnout: quando o trabalho ameaça o bem-estar do trabalhador. $4^{\circ}$ ed. São Paulo: Casa do Psicólogo, 2002; 282p.

31. PEREIRA MD, et al. Sofrimento emocional dos Enfermeiros no contexto hospitalar frente à pandemia de COVID-19. Research, Society and Development, 2020; 9(8): 1-21.

32. RAMOS RS. A Enfermagem Oncológica no Enfrentamento da Pandemia de Covid-19: Reflexões e Recomendações para a Prática de Cuidado em Oncologia. Revista Brasileira de Cancerologia, 2020; 66 (Tema atual): 1-5.

33. SAIDEL MGB, et al. Mental health interventions for health professionals in the context of the Coronavirus pandemic. Revista enfermagem UERJ, 2020; 28: 1-6.

34. SCHMIDT B, et al. Impactos na Saúde Mental e Intervenções Psicológicas Diante da Pandemia do Novo Coronavírus (COVID-19). Estudos de Psicologia, 2020; 37: 1-26.

35. SILVEIRA ALP, et al. Síndrome de Burnout: consequências e implicações de uma realidade cada vez mais prevalente na vida dos profissionais de saúde. Rev. Brasileira de Medicina do Trabalho, 2018; 14(3): 275-84.

36. TEIXEIRA FD, PREBIANCHI HB. Comprometimento, estresse e satisfação com a vida de profissionais da saúde. Revista Psicologia Organizações e Trabalho,2019; 15(4): 598-606.

37. TRIGO TR, et al. Síndrome de burnout ou estafa profissional e os transtornos psiquiátricos. Revista Psiquiatria Clínica, 2007; 34 (5): 223-233.

38. URQUIZA JLG, et al. Prevalence, risk factors, and levels of burnout among oncology nurses: A systematic review. Oncology Nursing Forum, 2016; 43(3): 104-120.

39. WORLD HEALTH ORGANIZATION (WHO). 2020. Coronavirus disease 2019 (COVID-19) Situation Report-78.

40. ZANATTA AB, LUCCA SR. Prevalência da síndrome de burnout em profissionais da saúde de um hospital oncohematológico infantil. Revista da Escola de Enfermagem da USP, 2015; 49(2): 253-260. 